\title{
Dose-response effect of Gelofusine on renal uptake and retention of radiolabelled octreotate in rats with CA20948 tumours
}

\author{
Marleen Melis • Magda Bijster • Monique de Visser • Mark W. Konijnenberg • \\ Jan de Swart • Edgar J. Rolleman • Otto C. Boerman • Eric P. Krenning • \\ Marion de Jong
}

Received: 10 March 2009 / Accepted: 3 June 2009/Published online: 8 July 2009

(C) The Author(s) 2009. This article is published with open access at Springerlink.com

\begin{abstract}
Purpose Peptide receptor radionuclide therapy using $\beta$ emitting radiolabelled somatostatin analogues like DOTA, $\mathrm{Tyr}^{3}$-octreotate shows beneficial results in patients suffering from somatostatin receptor overexpressing tumours. However, after high-dose therapy partial renal reabsorption of radiopeptides may lead to nephrotoxicity. Co-infusion of lysine/arginine lowers renal retention of these radiopeptides without affecting tumour uptake. Recently coadministration of Gelofusine has been described to have a comparable kidney-protecting effect in rats. In the present study optimal dosing of Gelofusine co-administration was studied in tumour-bearing rats.

Methods Doses of 40, 80, 120 or $160 \mathrm{mg} / \mathrm{kg}$ Gelofusine were co-injected with $15 \mu \mathrm{g}$ DOTA,Tyr ${ }^{3}$-octreotate, labelled with $3 \mathrm{MBq}{ }^{111} \mathrm{In}$ for biodistribution $(24 \mathrm{~h}$ postinjection, $n=4$ per group) and with $60 \mathrm{MBq}{ }^{111} \mathrm{In}$ for microSPECT imaging experiments at 3, 24 and $48 \mathrm{~h}$ postinjection. An additional group of rats received $80 \mathrm{mg} / \mathrm{kg}$ Gelofusine plus $400 \mathrm{mg} / \mathrm{kg}$ lysine co-injection. Biodis-
\end{abstract}

M. Melis $(\bowtie) \cdot$ M. Bijster $\cdot$ M. de Visser $\cdot$ J. de Swart

E. J. Rolleman $\cdot$ E. P. Krenning $\cdot$ M. de Jong

Department of Nuclear Medicine, Erasmus MC Rotterdam,

's Gravendijkwal 230,

3015 CE Rotterdam, The Netherlands

e-mail: m.melis@erasmusmc.nl

M. W. Konijnenberg

Research \& Development, Covidien,

Petten, The Netherlands

O. C. Boerman

Department of Nuclear Medicine, UMC St. Radboud,

Nijmegen, The Netherlands tribution studies were performed both in older (475 g) and younger $(300 \mathrm{~g})$ rats, the latter bearing CA20948 tumours.

Results Co-injection of $40 \mathrm{mg} / \mathrm{kg}$ Gelofusine resulted in $40-50 \%$ reduction of renal uptake and retention of ${ }^{111}$ In-DOTA, $\mathrm{Tyr}^{3}$-octreotate, whereas higher doses further increased the reduction to $50-60 \%$ in both groups of rats. Combining Gelofusine and lysine caused $70 \%$ reduction of renal uptake. The uptake of radiolabelled octreotate both in somatostatin receptor-expressing normal tissues and tumours was not affected by Gelofusine co-injection.

Conclusion In rats co-injection of $80 \mathrm{mg} / \mathrm{kg}$ Gelofusine resulted in maximum reduction of renal retention of ${ }^{111} \mathrm{In}$ DOTA, Tyr $^{3}$-octreotate, which was further improved when combined with lysine. Tumour uptake of radiolabelled octreotate was not affected, resulting in an increased tumour to kidney ratio.

Keywords Renal reabsorption - Radiolabelled octreotate Succinylated gelatin $\cdot$ Kidney

\section{Introduction}

The treatment of patients with somatostatin receptorpositive tumours with peptide receptor radionuclide therapy (PRRT) has shown convincing beneficial effects $[1,2]$. The radiolabelled peptides are rapidly cleared via the glomeruli in the kidneys into the urine, but a low percentage is reabsorbed and retained in the cortical proximal tubules $[3,4]$. After glomerular filtration a fraction of the administered peptides is internalized via endocytic receptors; in this process megalin has been demonstrated to play 
an essential role $[5,6]$. Transfer of the radiopeptides to the lysosomes is followed by degradation of the peptide, after which amino acid chelate conjugates are trapped in the lysosomes of the tubular cells [7], delivering a high radiation dose to the renal cortex during PRRT. The maximum tolerated dose in PRRT in patients is not exactly known. However, from data from external beam radiation therapy a limit of 23 Gy on the kidney has been adapted as the upper limit of the dose that can be administered safely [8]. Because of the differences with external beam irradiation individual dosimetry has been introduced recently in PRRT. This is to correct for the characteristic features of the radionuclide and of the patient, like inhomogeneous intra-organ distribution, shorter penetration range, dose rates following exponential decay and patientspecific geometry. Calculation of the biological equivalent dose (BED), with a correction for CT-assessed kidney volume and dose fractionation, has been adapted resulting in more accurate prediction of kidney toxicity. Using an upper limit of a BED of 40-45 Gy has been described to be safe to prevent nephrotoxicity during PRRT [9-11]. Coinfusion of lysine and arginine (Lys/Arg) has become a standard procedure in our institution during PRRT with ${ }^{177} \mathrm{Lu}$ - or ${ }^{90}$ Y-labelled somatostatin analogues, reducing the renal retention of the radiopeptides by approximately $35 \%$ [12-14]. These positively charged amino acids probably interfere in the megalin-mediated reabsorption process [4]. As a result higher radioactivity doses can be administered without risk of nephrotoxicity. In some cases, however, amino acid infusion may lead to nausea and vomiting, sometimes even to hyperkalaemia [13].

Recently, the reduction of renal retention of radiopeptides by co-infusion of the plasma expander Gelofusine was described $[15,16]$. A transient low molecular weight proteinuria $[17,18]$ induced by Gelofusine led to a $40 \%$ reduction of renal reabsorption of radiolabelled octreotide, both in animals $[15,19,20]$ and in humans [16]. The combination of both Lys and Gelofusine appeared to have an additive effect on the reduction of renal uptake of radiolabelled somatostatin analogues [19,20]. This pointed to different mechanisms of interfering in the reabsorption process in the renal proximal tubules.

Most of these experiments have been performed with a fixed dose of Gelofusine. The aim of the current study was to investigate the dose-response effect of Gelofusine on renal retention of the ${ }^{111}$ In-labelled somatostatin analogue DOTA, Tyr $^{3}$-octreotate. Biodistribution studies were performed in rats bearing CA20948 tumours expressing somatostatin receptors. Co-infusion of Lys has been described to have no effect on the tumour uptake of radiolabelled somatostatin analogues [21]; the effect of Gelofusine (with or without Lys) on receptor-mediated tumour uptake has not been described yet.

\section{Materials and methods}

Radionuclides, peptide, chemicals

${ }^{111} \mathrm{InCl}_{3}$ was purchased from Covidien (Petten, The Netherlands). DOTA, $\mathrm{Tyr}^{3}$-octreotate was obtained from BioSynthema (St. Louis, MO, USA). Radiolabelling was performed according to previously published procedures [22]. The labelling efficiency exceeded $99 \%$, as confirmed by thin-layer chromatography [22]. Specific activity of ${ }^{111} \mathrm{In}$ DOTA, Tyr ${ }^{3}$-octreotate was $3 \mathrm{MBq} / 15 \mu \mathrm{g}$ peptide for biodistribution studies and $60 \mathrm{MBq} / 15 \mu \mathrm{g}$ peptide for NanoSPECT imaging experiments. Gelofusine $(40 \mathrm{~g} / \mathrm{l})$ was obtained from Braun Medical (Oss, The Netherlands) and L-lysine from Sigma (Zwijndrecht, The Netherlands). Shortly before use a $400 \mathrm{mg} / \mathrm{ml} \mathrm{L}$-lysine solution in saline was prepared.

\section{Biodistribution experiments}

Animal studies were conducted in agreement with the Animal Welfare Committee requirements of our institution using generally accepted guidelines. For all experiments male Lewis rats (Harlan, Horst, The Netherlands) were used ( $n=4$ per group for biodistribution studies). A group of young, slim rats was used at 14 weeks of age (mean body weight of $300 \mathrm{~g}$ ) and a group of old, heavier rats at 45 weeks of age (mean body weight of $475 \mathrm{~g}$ ) at the time of biodistribution.

The young rats were subcutaneously inoculated with $500 \mu 1$ of a CA20948 tumour cell suspension [23], prepared from $5 \mathrm{~g}$ crude tumour tissue in $100 \mathrm{ml}$ saline, at both sides in the shoulder region. On day 24 after inoculation the biodistribution and imaging experiments were performed.

For biodistribution studies rats were anaesthetized with isoflurane $/ \mathrm{O}_{2}$ and injected with Gelofusine (or saline in control animals) intravenously via the tail vein. The volume ranged from 300 to $2,000 \mu \mathrm{l}$, depending on the administered dose ranging from 40 to $160 \mathrm{mg} / \mathrm{kg}$. In the experiment with the young rats one extra group received both $80 \mathrm{mg} / \mathrm{kg}$ Gelofusine and $400 \mathrm{mg} / \mathrm{kg}$ L-lysine. The injection of Gelofusine was immediately followed by the administration of ${ }^{111}$ In-DOTA,Tyr ${ }^{3}$-octreotate. A peptide dose of $15 \mu \mathrm{g}$ was used because this amount is required to administer the optimal activity dose of $555 \mathrm{MBq}{ }^{177} \mathrm{Lu}$-DOTA, $\mathrm{Tyr}^{3}$ octreotate in CA20948 tumour-bearing rats [21]. For the biodistribution studies $3 \mathrm{MBq}{ }^{111}$ In-DOTA,Tyr ${ }^{3}$-octreotate/ $15 \mu \mathrm{g}$ in $250 \mu \mathrm{l}$ was injected via the dorsal vein of the penis, whereas for imaging of the young tumour-bearing rats, one extra rat per group received $60 \mathrm{MBq}{ }^{111} \mathrm{In}$-DOTA, $\mathrm{Tyr}^{3}$-octreotate $/ 15 \mu \mathrm{g}$ in $250 \mu \mathrm{l}$.

After euthanasia at $24 \mathrm{~h}$ post-injection (p.i.), organs and tumours were dissected and blood samples were taken. Organs and tumours were weighed and radioactivity was 
measured in a gamma counter (Wallac, 1480 Wizard 3", PerkinElmer, Turku, Finland). Uptake of radioactivity was expressed as percentage of injected activity per gram tissue (\%IA/g). Data were expressed as percentage of control. For autoradiography one kidney and the tumours of each animal were frozen embedded in OCT compound (Tissue Tek, Sakura, Zoeterwoude, The Netherlands) using isopentane cooled in liquid nitrogen.

\section{MicroSPECT/CT imaging}

One additional rat from each group of the CA20948 tumour-bearing rats was imaged with the four-headed multi-pinhole NanoSPECT/CT camera (Bioscan Inc., Washington DC, USA) [24]. Rats were anaesthetized with isoflurane $/ \mathrm{O}_{2}$. Nine pinhole apertures with a diameter of $2.5 \mathrm{~mm}$ were used on each head, with a field of view (FOV) of $24 \mathrm{~mm}$. Settings of the ${ }^{111}$ In energy peaks were 171 and $245 \mathrm{keV}$. Based on the CT topogram, a body range of $85 \mathrm{~mm}$ ranging from neck to bottom was scanned in $24 \mathrm{~min}$ with $60 \mathrm{~s}$ per projection. A 6 min $\mathrm{CT}$ at $45 \mathrm{kV}_{\mathrm{p}}$ was acquired. Animals were imaged at 3, 24 and $48 \mathrm{~h}$ p.i. to measure the retention of ${ }^{111}$ In-DOTA, Tyr ${ }^{3}$-octreotate and allow calculation of the kidney and tumour dose.

Using the InVivoScope software quantification of the amount of radioactivity in the volume of interest (VOI) of kidneys and tumours was performed. Each tumour nodule inside a CA20948 tumour was analysed separately. The amount of radioactivity in the VOI was expressed in $\mathrm{MBq} / \mathrm{ml}$ tumour.

To achieve accurate quantification, the camera was calibrated by scanning a phantom, representing the attenuation of rats, filled with a known amount of ${ }^{111} \mathrm{In}$ activity.

\section{Ex vivo autoradiography}

Frozen kidneys and CA20948 tumours were cut into sections of $10 \mu \mathrm{m}$ (Microm Cryo-Star HM $560 \mathrm{M}$, Microm Laborgeräte GmbH, Walldorf, Germany). Autoradiographs of the sections were made by exposing them to SR phosphor imaging screens (PerkinElmer, Groningen, The Netherlands) in X-ray cassettes. After 24-72 $\mathrm{h}$ screens were read by a Cyclone phosphor imager and analysed with OptiQuant 03.00 image processing system (PerkinElmer, Groningen, The Netherlands). After exposure the sections were stained with haematoxylin and eosin (H\&E). Based on the results of this staining regions of interest (ROI) were determined. OptiQuant software was used to quantify the intensity of radioactivity and expressed in digital light units (DLU) $/ \mathrm{mm}^{2}$. Of each tumour five sections were analysed and at least five ROI per section.
Dosimetry

The dosimetry was based on the MIRD schema to calculate the dose by the product of the organ residence times and the $\mathrm{S}$ values for dose per cumulated activity. Radiation doses to the kidneys and CA20948 tumours were determined. The residence time of ${ }^{111} \mathrm{In}$ was determined by the kinetics of the radioactivity retention at three time points after administration. The kidney $\mathrm{S}$ values were calculated as described earlier [25], adapted to the actual mass of the kidneys, and the absorbed dose in $\mathrm{mGy} / \mathrm{MBq}$ was calculated. This dose was calculated separately for the whole kidney and for the cortex, because the majority of the radioactivity was retained in the cortex containing glomeruli and proximal tubules. Only the self-absorption dose from activity uptake in the kidneys was calculated. Tumour $\mathrm{S}$ values also were dependent on the actual mass of each tumour. Olinda/EXM software was used for calculation of the absorbed dose in $\mathrm{mGy} / \mathrm{MBq}[26]$.

\section{Statistics}

Data were expressed as mean \pm standard deviation (SD). Statistical analysis was performed using Student's $t$ test.

\section{Results}

In rat biodistribution studies the renal uptake of $15 \mu \mathrm{g}$ ${ }^{111}$ In-DOTA, Tyr $^{3}$-octreotate was examined, without or with co-injection of increasing doses of Gelofusine (ranging from 40 to $160 \mathrm{mg} / \mathrm{kg}$ ). The retained amount of radioactivity without Gelofusine co-administration was $4.6 \pm 0.3$ or $4.1 \pm 0.1 \% \mathrm{IA} / \mathrm{g}$ in the young and older rats, respectively, at $24 \mathrm{~h}$ p.i.. A dose of $40 \mathrm{mg} / \mathrm{kg}$ Gelofusine significantly reduced the renal retention of ${ }^{111}$ In-DOTA, $\mathrm{Tyr}^{3}$-octreotate (Fig. 1a, b). This lowest dose of Gelofusine induced a reduction of renal uptake of $40 \%$ in young and of $50 \%$ in older rats. When a dose of $80 \mathrm{mg} / \mathrm{kg}$ was given a further reduction of uptake in the kidneys was found of 50 and $60 \%$, respectively, compared to controls. With the 120 and $160 \mathrm{mg} / \mathrm{kg}$ Gelofusine doses no further reduction of the renal retention was measured; therefore, the $80 \mathrm{mg} / \mathrm{kg}$ dose seemed sufficient to induce the maximum achievable reduction of renal retention of ${ }^{111} \mathrm{In}$-DOTA, Tyr $^{3}$-octreotate. The maximum total volume of Gelofusine and radiopeptide that was administered to the heavy rats was more than $2,000 \mu \mathrm{l}$; no side effects were observed.

Ex vivo autoradiography of kidney sections demonstrated that the localization of the radioactivity was not affected by addition of Gelofusine (Fig. 1c); radioactivity mainly localized in the cortex and to a lesser extent in the outer 

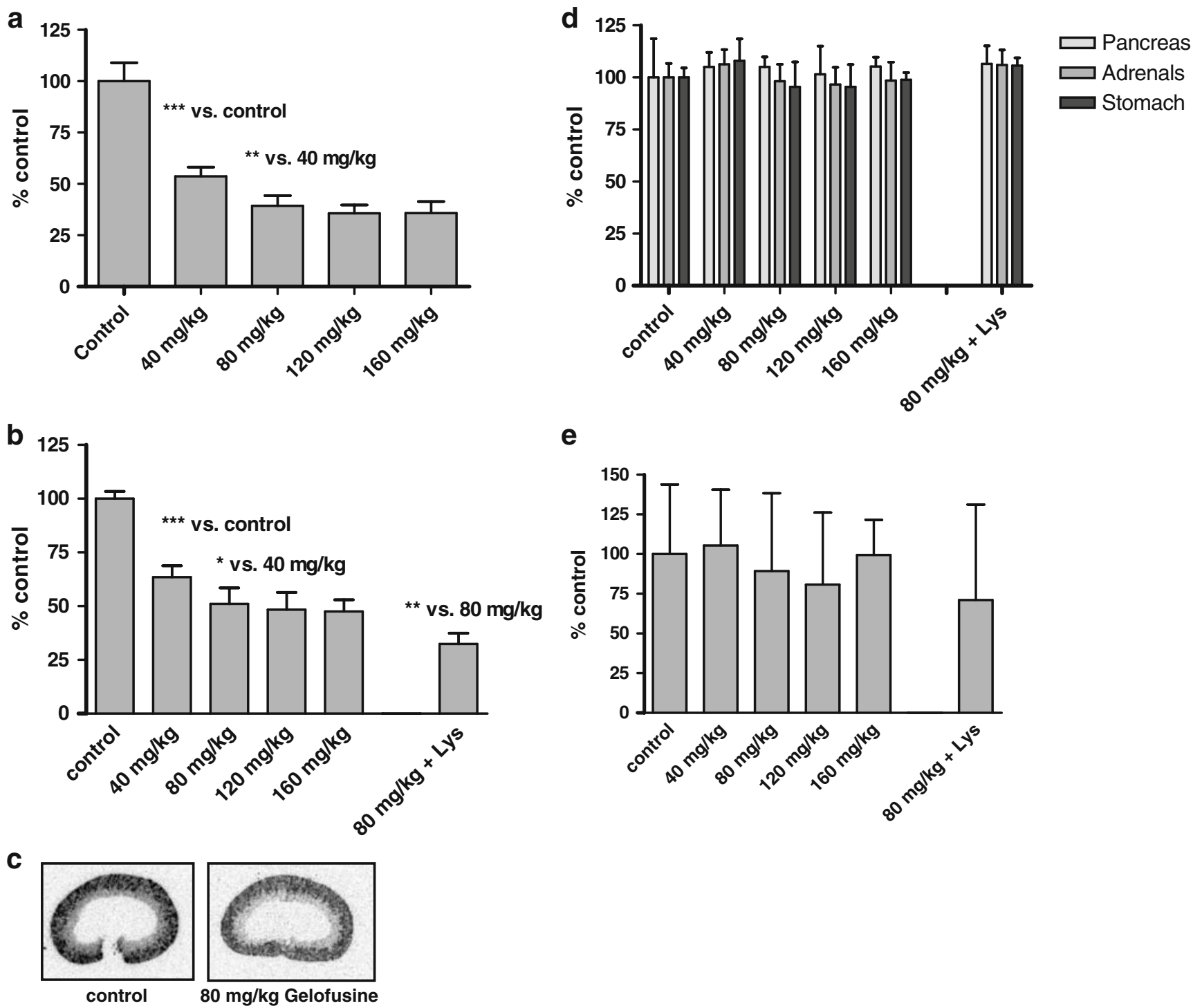

Fig. 1 Biodistribution at $24 \mathrm{~h}$ p.i. of $15 \mu \mathrm{g}{ }^{111}$ In-DOTA,Tyr ${ }^{3}$-octreotate in rats, without or with co-injection of Gelofusine in increasing doses ranging from 40 to $160 \mathrm{mg} / \mathrm{kg}$. In young rats Gelofusine $80 \mathrm{mg} / \mathrm{kg}$ in combination with Lys $400 \mathrm{mg} / \mathrm{kg}$ was tested as well. Uptake was calculated as $\%$ injected activity/gram $(\% \mathrm{IA} / \mathrm{g})$. The $\% \mathrm{IA} / \mathrm{g}$ of the control group was set at $100 \%$, and values obtained in Gelofusine groups were expressed as percentage of control values; $n=4$ per group. a Renal retention in 45-week-old rats with a mean body weight of

475 g. b Renal retention in 14-week- old rats with a mean body weight of 300 g. c Kidney sections after ex vivo autoradiography demonstrating localization of ${ }^{111} \mathrm{In}$-DOTA, Tyr ${ }^{3}$-octreotate $24 \mathrm{~h}$ p.i. in a representative kidney from the control and Gelofusine $80 \mathrm{mg} / \mathrm{kg}$ group of the 45-week-old rats. d Uptake in pancreas, adrenals and stomach in 14-week-old rats. e Uptake in CA20948 tumours in 14-week-old rats. ${ }^{*} p<0.05 ;{ }^{* *} p<0.001 ;{ }^{* * *} p<0.005$

medulla. When the $80 \mathrm{mg} / \mathrm{kg}$ Gelofusine dose was combined with Lys co-administration a significant additive effect on reduction of renal uptake of radiolabelled octreotate was measured; $68 \%$ reduction compared to control values was achieved (Fig. 1b). In the biodistribution study in the young rats it was found that the administration of large amounts of Gelofusine, with or without Lys, did not affect the uptake of ${ }^{111}$ In-DOTA, Tyr ${ }^{3}$-octreotate in somatostatin receptor-expressing organs (Fig. 1d) and subcutaneously grown somatostatin receptor-expressing

CA20948 pancreatic tumours (Fig. 1e). Quantification of retained radioactivity in CA20948 tumour sections in ex vivo autoradiograms confirmed that Gelofusine coadministration had no significant effect on tumour uptake of radiolabelled octreotate (Fig. 2a, b).

Tumour to kidney activity uptake ratios at $24 \mathrm{~h}$ p.i. of the control, the Gelofusine $80 \mathrm{mg} / \mathrm{kg}$ and the combination of Gelofusine and Lys situation were $0.18 \pm 0.08,0.30 \pm 0.12$ and $0.38 \pm 0.23$, respectively. This was also nicely visualized in the NanoSPECT/CT images of CA20948 tumour- 


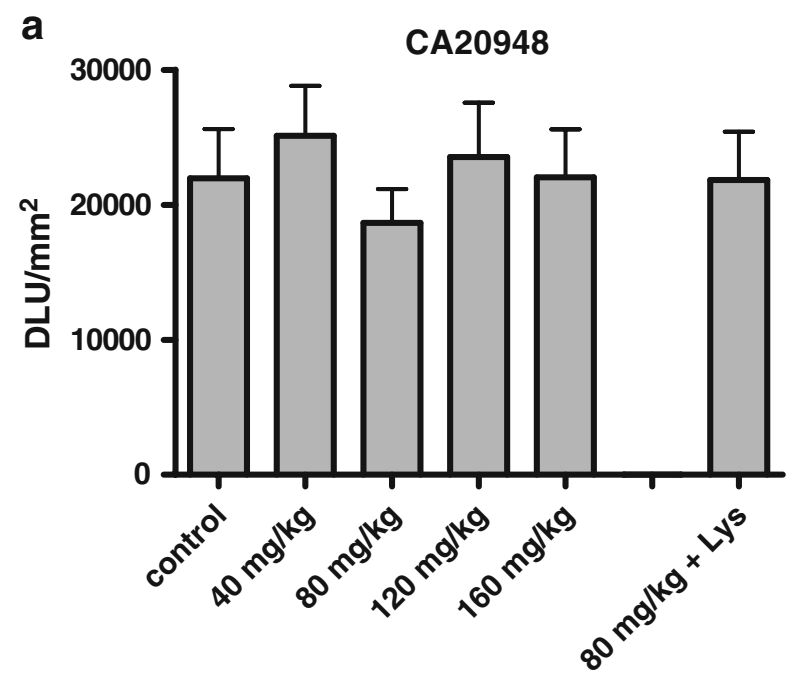

b

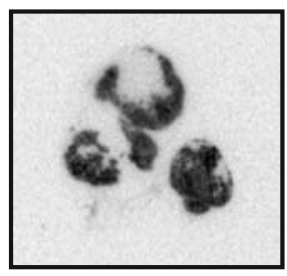

Control

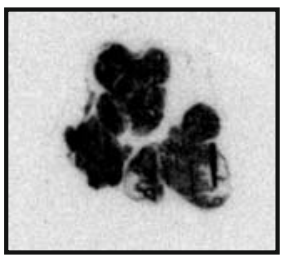

$40 \mathrm{mg} / \mathrm{kg}$

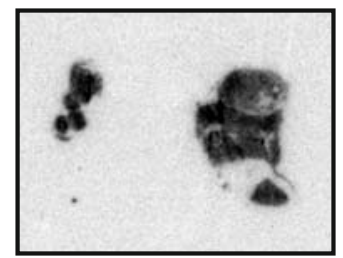

$80 \mathrm{mg} / \mathrm{kg}$
Fig. 2 Ex vivo autoradiography of CA20948 after biodistribution study with ${ }^{111}$ In-DOTA,Tyr ${ }^{3}$-octreotate, without or with co-injection of increasing doses of Gelofusine. a Quantification of intensity of retained radioactivity in frozen sections of CA20948 tumours after ex vivo autoradiography of which the biodistribution results are shown in

bearing rats (Fig. 3a) [27]. Quantification of the tumour uptake of ${ }^{111}$ In-DOTA, Tyr $^{3}$-octreotate $24 \mathrm{~h}$ p.i. in the images revealed again that increasing doses of Gelofusine did not affect tumour uptake of radiolabelled octreotate expressed in $\mathrm{MBq} / \mathrm{ml}$ tumour (Fig. 3b).

Images of the rats acquired at 3,24 and $48 \mathrm{~h}$ p.i. were analysed to determine the kidney and tumour dosimetry. The kinetics of the washout of ${ }^{111}$ In-DOTA,Tyr ${ }^{3}$-octreotate from the kidneys and CA20948 tumours were similar in the control group and the Gelofusine (+ Lys) groups; kidney data are shown in Fig. 3c. After determination of the correct $\mathrm{S}$ values based on the actual masses of both kidneys and tumours, the absorbed doses were calculated. The dose on the renal cortex in the rats that received Gelofusine $80 \mathrm{mg} / \mathrm{kg}$ appeared to be $40 \%$ of the dose in the rats without renal protection, whereas the dose in the rats that received the combination of Gelofusine with Lys was only $30 \%$ of the dose in the control group as shown in Fig. 3d. The total absorbed radiation dose caused by ${ }^{111} \mathrm{In}$ to the renal cortex in the control group was $1.7 \pm 0.1 \mathrm{~Gy}$, whereas the doses to the CA20948 tumours varied between 0.3 and $0.75 \mathrm{~Gy}$ and were independent of administration of renal protection agents.

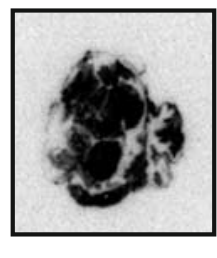

$120 \mathrm{mg} / \mathrm{kg}$

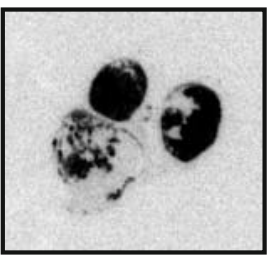

$160 \mathrm{mg} / \mathrm{kg}$

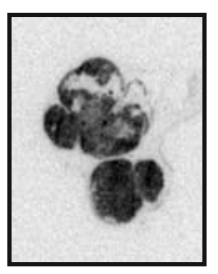

$80 \mathrm{mg} / \mathrm{kg}+$ Lys
Fig. 1e. Amount of radioactivity is expressed in DLU/mm². Only regions containing tumour cells were analysed, as demonstrated in the H\&E-stained section. No significant differences were found. b Examples of CA20948 tumour sections after ex vivo autoradiography

\section{Discussion}

Reduction of renal retention of radiolabelled somatostatin analogues is an important issue in PRRT, since after treatment with therapeutic doses of ${ }^{90}$ Y-labelled octreotide or octreotate nephrotoxicity has been described [28, 29], especially when renal doses exceeding 23 Gy were applied $[14,30]$. The partial reabsorption of somatostatin analogues can be reduced by co-infusion with the cationic amino acids lysine and arginine during the administration of therapeutic doses of ${ }^{90} \mathrm{Y}$ - or ${ }^{177} \mathrm{Lu}$-labelled somatostatin analogues. This is a commonly used method nowadays, resulting in about $35 \%$ reduction of renal uptake of radioactivity $[2,13]$. Approximately $30 \%$ of the patients suffer from nausea and $15 \%$ from vomiting as well during the $4 \mathrm{~h}$ infusion of $2.5 \%$ lysine and $2.5 \%$ arginine in 11 of saline, in spite of administration of the antiemetic granisetron [31]. Hyperkalaemia has not been demonstrated with these amounts of amino acids, but was only found in patients receiving a total dose of $75 \mathrm{~g}$ lysine [13]. Therefore, increasing the Lys dose is not an option to improve the reduction of renal uptake. Research to further reduce renal retention of such high-energy radiolabelled 


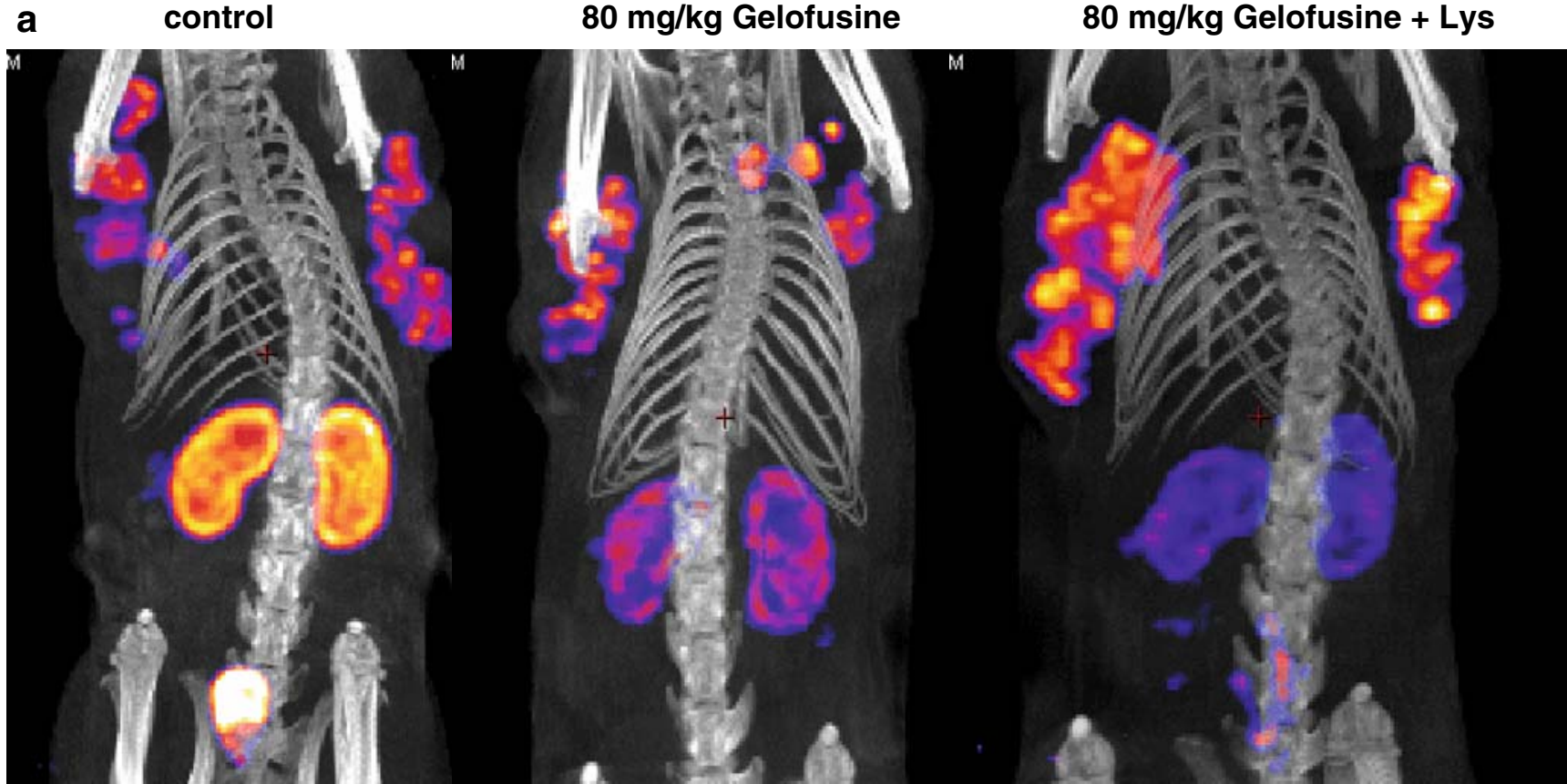

b

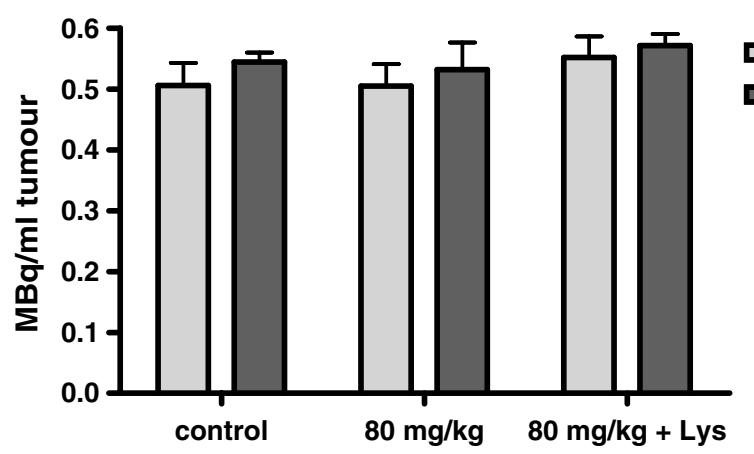

d

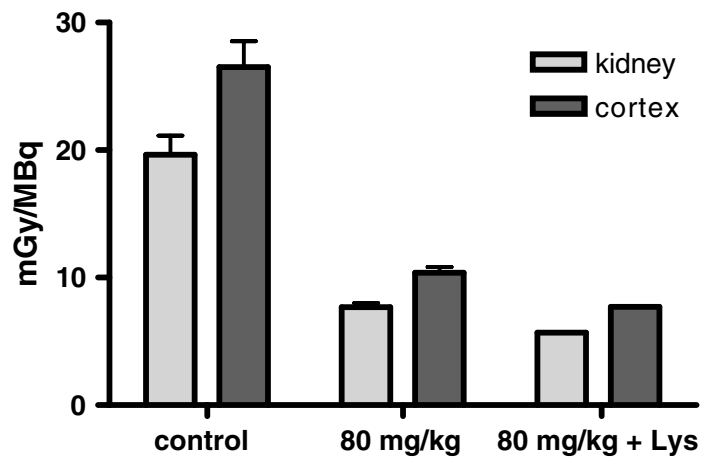

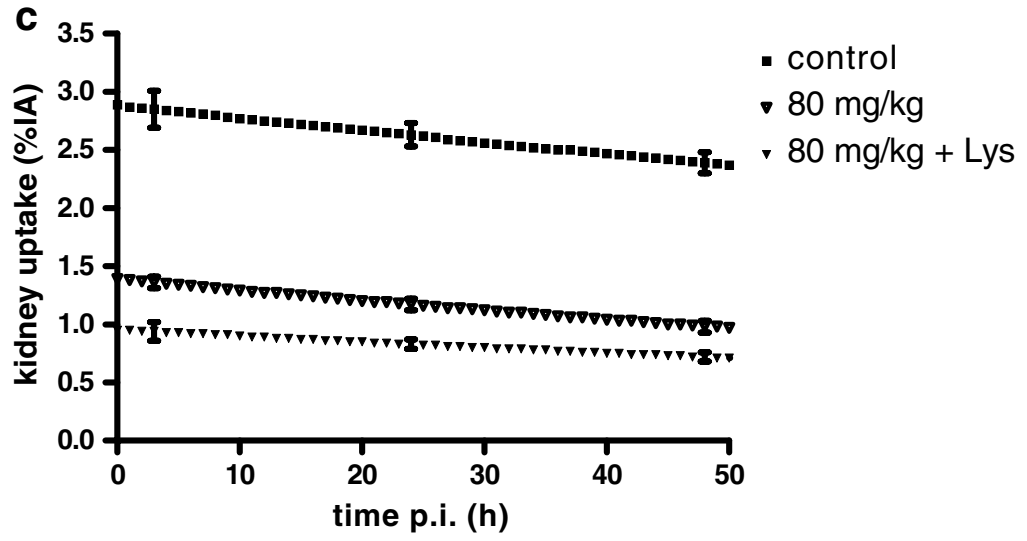

Fig. 3 NanoSPECT/CT imaging, quantification and dosimetry. a NanoSPECT/CT images of CA20948-tumour bearing rats, $3 \mathrm{~h}$ p.i. of $15 \mu \mathrm{g}{ }^{111}$ In-DOTA,Tyr ${ }^{3}$-octreotate, labelled with $60 \mathrm{MBq}$ of ${ }^{111} \mathrm{In}$, without or with co-injection of Gelofusine $80 \mathrm{mg} / \mathrm{kg}$ (and $400 \mathrm{mg} / \mathrm{kg}$ Lys). Earlier published in [27]. b Quantification of retained radioactivity in CA20948 tumours of imaged rats using InVivoScope software. Amount of radioactivity was expressed in $\mathrm{MBq} / \mathrm{ml}$ tumour. Each tumour nodule inside a CA20948 tumour was analysed separately. No significant differences were found. $\mathbf{c}$ Residence time of ${ }^{111} \mathrm{In}$ in kidneys, as determined in NanoSPECT/CT images at 3, 24 and $48 \mathrm{~h}$ p.i. of $15 \mu \mathrm{g}$ ${ }^{111}$ In-DOTA, Tyr $^{3}$-octreotate. Washout of ${ }^{111}$ In was plotted for three rats: control, with Gelofusine $80 \mathrm{mg} / \mathrm{kg}$ alone and combined with $400 \mathrm{mg} / \mathrm{kg}$ Lys. d Dose calculation of ${ }^{111}$ In-DOTA, Tyr ${ }^{3}$-octreotate for whole kidney or renal cortex only, expressed in $\mathrm{mGy} / \mathrm{MBq}{ }^{111} \mathrm{In}$. Renal radiation dose in a control rat is compared with rats receiving Gelofusine $80 \mathrm{mg} / \mathrm{kg}$ alone or combined with $400 \mathrm{mg} / \mathrm{kg}$ Lys as co-administration 
peptides is warranted to increase the therapeutic window of PRRT.

The net charge of radiolabelled peptides is an important factor influencing renal retention [32]. The Lys residue in octreotide and octreotate seems responsible for the relatively high uptake in kidney. In a preclinical study comparing different neurotensin analogues it was shown that Lys coadministration also reduced renal retention of other radiolabelled peptides, but this only occurred when a Lys residue formed part of the peptide [33]. This points to a competitive mechanism which has been revealed to be, at least partly, a megalin-mediated process. This negatively charged multiligand scavenger receptor which is expressed on renal proximal tubules plays a key role in the endocytosis of radiolabelled somatostatin analogues $[4,6]$.

Gelofusine is a succinylated gelatine consisting of polypeptides with an average molecular weight of $30 \mathrm{kDa}$. It is used as a plasma expander in critically ill patients. ten Dam et al. described that after Gelofusine infusion an increased amount of $\alpha_{1}$ - and $\beta_{2}$-microglobulin was excreted in the urine, pointing to a disturbed protein reabsorption process [17]. Based on this observation Gelofusine was coadministered with radiolabelled somatostatin analogues and offered a reduction of renal uptake of radiolabelled octreotide to an extent comparable to that of cationic amino acids, as recently described in animals and in healthy volunteers $[15,16]$. The exact mechanism of the reducing effect of Gelofusine is still unknown. When the renal uptake of several non-somatostatin peptide analogues was studied, it appeared that their renal retention could be reduced as well, whereas in the cases of gastrin and exendin analogues Lys co-injection had no effect [19, 34]. Gotthardt et al. suggested that Lys and polyglutamic acid only interfere with one of the four clusters of anionic amino acid repeats of megalin responsible for binding of a variety of ligands, while Gelofusine administration acts on all four clusters enabling reduced retention of all radiolabelled peptides tested thus far [19, 35].

To further reduce uptake and retention of radioactivity in the kidneys the combination of both agents was tested, demonstrating an additive effect. This combination of agents is promising, because more cycles of therapeutic doses could be safely administered in clinical PRRT using this combination without exceeding the mentioned 23 Gy absorbed kidney dose or 45 Gy BED limit. Until now in most experiments in rats and mice a fixed dose of Gelofusine was used $[15,19]$. In the study of Rolleman et al. [20], a limited dose-response effect was suggested in the range between 50 and $80 \mathrm{mg} / \mathrm{kg}$. In the current study this observation was confirmed; $40 \mathrm{mg} / \mathrm{kg}$ Gelofusine already caused a reduction of renal retention of ${ }^{111}$ In-DOTA, $\mathrm{Tyr}^{3}$ octreotate, which was further increased with a $80 \mathrm{mg} / \mathrm{kg}$ dose. These doses were independent of the body weight of the animals, since comparable results were obtained in both groups without significant difference. The $80 \mathrm{mg} / \mathrm{kg}$ dose appeared to be the dose being sufficient to induce maximum reduction of renal retention, since higher doses did not improve the reduction of kidney uptake of radiolabelled octreotate. Apparently maximal induction of proteinuria and/or blocking of receptors responsible for endocytosis already is achieved at this $80 \mathrm{mg} / \mathrm{kg}$ Gelofusine dose.

Comparing literature data with the currently described results, it is clear that the percentage of reduction of renal uptake by Gelofusine co-administration in a $80 \mathrm{mg} / \mathrm{kg}$ dose is comparable for all somatostatin analogues tested $\left({ }^{111}\right.$ In-DTPA, $\mathrm{Tyr}^{3}$-octreotide, ${ }^{177} \mathrm{Lu}$-DOTA,Tyr ${ }^{3}$-octreotate, ${ }^{111}$ In-DOTA, $\mathrm{Tyr}^{3}$-octreotate) and is independent of the administered amount of peptide $(0.5 \mu \mathrm{g}, 15 \mu \mathrm{g})$, while the \%IA/g kidney of control animals ranged from 1 to $4 \% \mathrm{IA} / \mathrm{g}[15,19,20]$.

The potential effect of Gelofusine on tumour uptake of ${ }^{111}$ In-DOTA, Tyr ${ }^{3}$-octreotate was investigated as well. The biodistribution experiment showed no interference of Gelofusine with radioactivity uptake in CA20948 tumour, although the tumour uptake varied largely due to the irregular shapes and structures of the tumours, whereas the mean volume of the tumours was similar in all groups, although with a high variability. When radioactivity in separate tumour nodules in the frozen sections was quantified using ex vivo autoradiography, variability of tumour uptake was smaller and mean DLU/ $\mathrm{mm}^{2}$ was not reduced when Gelofusine was co-administered. Furthermore, the recently developed method to quantify the amount of radioactivity which is retained in imaged organs and tumours using microSPECT/CT also confirmed that tumour uptake of ${ }^{111}$ In-DOTA, Tyr $^{3}$-octreotate was not influenced by Gelofusine with or without Lys [20, 24].

Quantification of the retained renal radioactivity by imaging the same rat at several time points after injection of the radiolabelled octreotate allowed estimation of the radiation dose to the kidneys and tumours. The clearance rate of ${ }^{111}$ In-DOTA, Tyr ${ }^{3}$-octreotate from the kidneys and the tumours was similar in the control and in the renal protection groups. This confirms that Gelofusine and Lys had a transient effect on the renal reabsorption mechanism and only interfered in the initial retention of radiopeptides in the proximal tubules, but had no effect on the washout of the radiometals. The induced reduction in radiation dose to the whole kidney or to cortex alone was comparable to the reduction found in the biodistribution study: up to $70 \%$, which is very favourable for kidney protection when therapeutic radionuclides like ${ }^{177} \mathrm{Lu}$ or ${ }^{90} \mathrm{Y}$ will be applied.

Taken together our results indicating significant renal protection but unaffected uptake in somatostatin receptorexpressing tumours and organs warrant a randomized cross- 
over trial to test the combination of amino acid infusions with or without Gelofusine in patients receiving PRRT with ${ }^{177}$ Lu-DOTA, Tyr $^{3}$-octreotate in our centre.

It is hard to translate our reported optimal dose of $80 \mathrm{mg} / \mathrm{kg}$ Gelofusine for renal uptake reduction of ${ }^{111} \mathrm{In}$ DOTA, Tyr $^{3}$-octreotate in rats, which was administered as a bolus injection, to the clinical situation where Gelofusine will be co-infused during several hours. The Gelofusine dose that was administered in the healthy volunteers was a bolus of $40 \mathrm{mg} / \mathrm{kg}$ per $10 \mathrm{~min}$ followed by a $3 \mathrm{~h}$ infusion of $0.8 \mathrm{mg} / \mathrm{kg}$ per $\min (144 \mathrm{mg} / \mathrm{kg})$, resulting in a total dose of almost $200 \mathrm{mg} / \mathrm{kg}$ over a 3 -h period of time [16]. In Bad Berka $450 \mathrm{ml}$ of $4 \%$ Gelofusine solution was infused in $4 \mathrm{~h}$ combined with Lys/Arg, during PRRT, which means a dose of $225 \mathrm{mg} / \mathrm{kg}$ per $4 \mathrm{~h}$ for a patient with a body weight of $80 \mathrm{~kg}$ [36].

A drawback of the use of Gelofusine might be the risk of an allergic reaction in less than $1 \%$ of the patients, probably because of its bovine origin $[37,38]$. Therefore, patients need to be strictly monitored. When a significantly reduced renal retention of radioactivity can be obtained with extra Gelofusine administration, an adapted regimen to protect kidneys from renal toxicity during PRRT may be used in future. This will allow administration of more cycles of PRRT without risk of nephrotoxicity, while a higher tumour radiation dose will be achieved.

Due to crossfire from circulating radioactivity in the blood the bone marrow is the second organ at risk during PRRT. The clearance of radioactivity from the blood was not delayed by the administration of Gelofusine and Lys. Therefore, especially when the cumulative dose will be increased further, monitoring of blood cell counts is needed to detect haematological toxicity, like cytopaenia or myelodysplastic syndrome [2].

\section{Conclusion}

In rats a Gelofusine dose of $80 \mathrm{mg} / \mathrm{kg}$ body weight induced maximal reduction of $50-60 \%$ of renal retention after administration of a therapeutic peptide dose of $15 \mu \mathrm{g}$ of ${ }^{111}$ In-DOTA, $\mathrm{Tyr}^{3}$-octreotate. Combination of this dose of Gelofusine with $400 \mathrm{mg} / \mathrm{kg}$ L-lysine resulted in a $70 \%$ reduced kidney uptake, while the somatostatin receptor-specific uptake in pancreas, stomach, adrenals and CA20948 pancreatic tumour was not affected. Application of combined Lys/Arg and Gelofusine infusions during PRRT in patients will enlarge the therapeutic window.

Acknowledgements This study was supported by KWF/NKB (Dutch Cancer Foundation), grant no. EMCR 2007-3758. Technical assistance of Erik de Blois and Walter Spreeuwenberg was greatly appreciated. Collaboration within COST working group BM0607 contributed to stimulating discussions.
Open Access This article is distributed under the terms of the Creative Commons Attribution Noncommercial License which permits any noncommercial use, distribution, and reproduction in any medium, provided the original author(s) and source are credited.

\section{References}

1. Krenning EP, Teunissen JJ, Valkema R, deHerder WW, deJong M, Kwekkeboom DJ. Molecular radiotherapy with somatostatin analogs for (neuro-)endocrine tumors. $J$ Endocrinol Invest 2005;28(11 Suppl International):146-50.

2. Kwekkeboom DJ, de Herder WW, Kam BL, van Eijck CH, van Essen M, Kooij PP, et al. Treatment with the radiolabeled somatostatin analog [177 Lu-DOTA 0, Tyr3]octreotate: toxicity, efficacy, and survival. J Clin Oncol 2008;26(13):2124-30.

3. Silbernagl S. The renal handling of amino acids and oligopeptides. Physiol Rev 1988;68(3):911-1007.

4. Melis M, Krenning EP, Bernard BF, Barone R, Visser TJ, de Jong $\mathrm{M}$. Localisation and mechanism of renal retention of radiolabelled somatostatin analogues. Eur J Nucl Med Mol Imaging 2005;32 (10):1136-43.

5. Christensen EI, Birn H. Megalin and cubilin: multifunctional endocytic receptors. Nat Rev Mol Cell Biol 2002;3(4):256-66.

6. de Jong M, Barone R, Krenning E, Bernard B, Melis M, Visser T, et al. Megalin is essential for renal proximal tubule reabsorption of (111)In-DTPA-octreotide. J Nucl Med 2005;46(10):1696-700.

7. Duncan JR, Welch MJ. Intracellular metabolism of indium-111DTPA-labeled receptor targeted proteins. J Nucl Med 1993;34 (10):1728-38.

8. Emami B, Lyman J, Brown A, Coia L, Goitein M, Munzenrider $\mathrm{JE}$, et al. Tolerance of normal tissue to therapeutic irradiation. Int $\mathrm{J}$ Radiat Oncol Biol Phys 1991;21(1):109-22.

9. Barone R, Borson-Chazot F, Valkema R, Walrand S, Chauvin F, Gogou L, et al. Patient-specific dosimetry in predicting renal toxicity with (90)Y-DOTATOC: relevance of kidney volume and dose rate in finding a dose-effect relationship. J Nucl Med 2005;46(Suppl 1):99S-106S.

10. Bodei L, Cremonesi M, Ferrari M, Pacifici M, Grana CM, Bartolomei $\mathrm{M}$, et al. Long-term evaluation of renal toxicity after peptide receptor radionuclide therapy with 90Y-DOTATOC and 177Lu-DOTATATE: the role of associated risk factors. Eur J Nucl Med Mol Imaging 2008;35(10):1847-56.

11. Wessels BW, Konijnenberg MW, Dale RG, Breitz HB, Cremonesi M, Meredith RF, et al. MIRD pamphlet No. 20: the effect of model assumptions on kidney dosimetry and response-implications for radionuclide therapy. J Nucl Med 2008;49(11):1884-99.

12. de Jong M, Rolleman EJ, Bernard BF, Visser TJ, Bakker WH, Breeman WA, et al. Inhibition of renal uptake of indium-111DTPA-octreotide in vivo. J Nucl Med 1996;37(8):1388-92.

13. Rolleman EJ, Valkema R, de Jong M, Kooij PP, Krenning EP. Safe and effective inhibition of renal uptake of radiolabelled octreotide by a combination of lysine and arginine. Eur J Nucl Med Mol Imaging 2003;30(1):9-15.

14. Valkema R, Pauwels SA, Kvols LK, Kwekkeboom DJ, Jamar F, de Jong $\mathrm{M}$, et al. Long-term follow-up of renal function after peptide receptor radiation therapy with (90)Y-DOTA(0),Tyr(3)octreotide and (177)Lu-DOTA(0), Tyr(3)-octreotate. J Nucl Med 2005;46(Suppl 1):83S-91S.

15. van Eerd JE, Vegt E, Wetzels JF, Russel FG, Masereeuw R, Corstens FH, et al. Gelatin-based plasma expander effectively reduces renal uptake of $111 \mathrm{In}$-octreotide in mice and rats. J Nucl Med 2006;47(3):528-33. 
16. Vegt E, Wetzels JF, Russel FG, Masereeuw R, Boerman OC, van Eerd JE, et al. Renal uptake of radiolabeled octreotide in human subjects is efficiently inhibited by succinylated gelatin. J Nucl Med 2006;47(3):432-6.

17. ten Dam MA, Branten AJ, Klasen IS, Wetzels JF. The gelatinderived plasma substitute Gelofusine causes low-molecularweight proteinuria by decreasing tubular protein reabsorption. $\mathrm{J}$ Crit Care 2001;16(3):115-20.

18. Veldman BA, Schepkens HL, Vervoort G, Klasen I, Wetzels JF. Low concentrations of intravenous polygelines promote low-molecular weight proteinuria. Eur J Clin Invest 2003;33(11):962-8.

19. Gotthardt M, van Eerd-Vismale J, Oyen WJ, de Jong M, Zhang H, Rolleman E, et al. Indication for different mechanisms of kidney uptake of radiolabeled peptides. J Nucl Med 2007;48(4):596-601.

20. Rolleman EJ, Bernard BF, Breeman WA, Forrer F, de Blois E, Hoppin $\mathrm{J}$, et al. Molecular imaging of reduced renal uptake of radiolabelled [DOTA0, Tyr3] octreotate by the combination of lysine and Gelofusine in rats. Nuklearmedizin 2008;47(3):110-5.

21. de Jong M, Breeman WA, Bernard BF, Bakker WH, Schaar M, van Gameren A, et al. [177Lu-DOTA(0), Tyr3] octreotate for somatostatin receptor-targeted radionuclide therapy. Int $\mathrm{J}$ Cancer 2001;92(5):628-33.

22. Breeman WA, De Jong M, Visser TJ, Erion JL, Krenning EP. Optimising conditions for radiolabelling of DOTA-peptides with 90Y, 111 In and 177Lu at high specific activities. Eur J Nucl Med Mol Imaging 2003;30(6):917-20.

23. Bernard BF, Krenning E, Breeman WA, Visser TJ, Bakker WH, Srinivasan A, et al. Use of the rat pancreatic CA20948 cell line for the comparison of radiolabelled peptides for receptor-targeted scintigraphy and radionuclide therapy. Nucl Med Commun 2000;21(11):1079-85.

24. Forrer F, Valkema R, Bernard B, Schramm NU, Hoppin JW, Rolleman $\mathrm{E}$, et al. In vivo radionuclide uptake quantification using a multi-pinhole SPECT system to predict renal function in small animals. Eur J Nucl Med Mol Imaging 2006;33(10):1214-7.

25. Konijnenberg MW, Bijster M, Krenning EP, De Jong M. A stylized computational model of the rat for organ dosimetry in support of preclinical evaluations of peptide receptor radionuclide therapy with (90)Y, (111)In, or (177)Lu. J Nucl Med 2004;45(7):1260-9.

26. Stabin MG, Konijnenberg MW. Re-evaluation of absorbed fractions for photons and electrons in spheres of various sizes. J Nucl Med 2000;41(1):149-60.
27. Schober O, Rahbar K, Riemann B. Multimodality molecular imaging - from target description to clinical studies. Eur J Nucl Med Mol Imaging 2009;36(2):302-14.

28. Otte A, Herrmann R, Heppeler A, Behe M, Jermann E, Powell P, et al. Yttrium-90 DOTATOC: first clinical results. Eur J Nucl Med 1999;26(11):1439-47.

29. Cybulla M, Weiner SM, Otte A. End-stage renal disease after treatment with 90Y-DOTATOC. Eur J Nucl Med 2001;28 (10):1552-4.

30. Bodei L, Cremonesi M, Grana C, Rocca P, Bartolomei M, Chinol $\mathrm{M}$, et al. Receptor radionuclide therapy with 90Y-[DOTA]0-Tyr3octreotide (90Y-DOTATOC) in neuroendocrine tumours. Eur $\mathrm{J}$ Nucl Med Mol Imaging 2004;31(7):1038-46.

31. Kwekkeboom DJ, Teunissen JJ, Bakker WH, Kooij PP, de Herder WW, Feelders RA, et al. Radiolabeled somatostatin analog [177LuDOTA0, Tyr3]octreotate in patients with endocrine gastroenteropancreatic tumors. J Clin Oncol 2005;23(12):2754-62.

32. Akizawa H, Arano Y, Mifune M, Iwado A, Saito Y, Mukai T, et al. Effect of molecular charges on renal uptake of 111In-DTPAconjugated peptides. Nucl Med Biol 2001;28(7):761-8.

33. Janssen PJ, de Visser M, Verwijnen SM, Bernard BF, Srinivasan A, Erion JL, et al. Five stabilized 111In-labeled neurotensin analogs in nude mice bearing HT29 tumors. Cancer Biother Radiopharm 2007;22(3):374-81.

34. Knör S, Sato S, Huber T, Morgenstern A, Bruchertseifer F, Schmitt M, et al. Development and evaluation of peptidic ligands targeting tumour-associated urokinase plasminogen activator receptor (UPAR) for use in alpha-emitter therapy for disseminated ovarian cancer. Eur J Nucl Med Mol Imaging 2008;35(1):53-64.

35. Nagai J, Takano M. Molecular aspects of renal handling of aminoglycosides and strategies for preventing the nephrotoxicity. Drug Metab Pharmacokinet 2004;19(3):159-70.

36. Prasad V, Fetscher S, Baum RP. Changing role of somatostatin receptor targeted drugs in NET: Nuclear Medicine's view. J Pharm Pharm Sci 2007;10(2):321s-37s.

37. Barron ME, Wilkes MM, Navickis RJ. A systematic review of the comparative safety of colloids. Arch Surg 2004;139(5):552-63.

38. Rolleman EJ, de Jong M, Valkema R, Kwekkeboom D, Kam B, Krenning EP. Inhibition of kidney uptake of radiolabeled somatostatin analogs: amino acids or gelofusine? J Nucl Med 2006;47(10):1730-1. author reply 1731 . 